\title{
SIADH as the initial manifestation of acute intermittent porphyria: A case report
}

\author{
Tony K S' ${ }^{1}$, Rakhee Joshi ${ }^{2}$, Pradnya Chaudhari ${ }^{3}$, Reshma Abraham ${ }^{1}$, Nachiket Joshi ${ }^{1}$, Adwait Mulye ${ }^{1}$, \\ Abhinav Wankhede ${ }^{1}$ \\ From ${ }^{1}$ Junior Resident, ${ }^{2}$ Associate Professor, ${ }^{3}$ Lecturer, Department of General Medicine, Indira Gandhi Government Medical College, Nagpur, \\ Maharashtra, India
}

\begin{abstract}
Acute intermittent porphyria (AIP) is a rare autosomal dominant and the most severe form of the inherited hepatic porphyrias, affecting mainly young women. We present the case of an 18-year-old female who presented with severe abdominal pain, purple urine, autonomic dysfunction, and severe hyponatremia, seizures on further evaluation came out to be a case of AIP. This case report is a reminder to keep AIP among the differentials in young female patients with a classic constellation of abdominal urine discoloration syndrome of inappropriate secretion of antidiuretic hormone and autonomic dysfunction.
\end{abstract}

Key words: Acute intermittent porphyria, Hyponatremia, Porphobilinogen, Porphyria

$\mathrm{T}$ here are four acute porphyrias, namely, $\delta$-aminolevulinic acid (ALA) dehydratase deficiency porphyria (ADP), acute intermittent porphyria (AIP), hereditary coproporphyria (HCP), and variegate porphyria (VP), all of which are inheritable metabolic diseases of heme synthesis and all of them are hepatic porphyrias. AIP, HCP, and VP are all autosomal dominant inherited with low penetrance and share the common characteristic of by-episodic occurrence of life-threatening acute neurovisceral attacks, which are identical in all three conditions. ADP is inherited autosomal recessive manner and is very rare. In VP and HCP, bullous skin lesions may occur with or apart from acute attacks. Skin lesions are much more common in VP than in HCP [1]. AIP is also known as Swedish porphyria, pyrroloporphyria, or intermittent acute porphyria [1]. The overall prevalence of clinically manifest AIP is estimated to be $50-500$ /million [2]. AIP is caused by deficiency of hydroxymethylbilane synthase or porphobilinogen (PBG) deaminase enzyme (third enzyme in the pathway of heme synthesis) which in turn causes increased ALA and PBG [3]. Diagnosis is often delayed as the symptoms are nonspecific. The syndrome of inappropriate secretion of antidiuretic hormone (SIADH) is a rare manifestation of AIP and very only a few cases have been reported [4].

\section{CASE REPORT}

An 18-year-old female patient presented with complaints of severe colicky abdominal pain around her umbilicus about

\section{Access this article online}

Received - 18 October 2021

Initial Review - 03 November 2021

Accepted - 11 November 2021

DOI: $10.32677 /$ ijer.v7i11.3114
6 days after the menstrual period. The pain was continuous with associated palpitation, vomiting, and constipation for 3 days. The patient was also complaining of discoloration of urine along with such episodes since childhood. She presented to the casualty on the 2 day of abdominal pain.

On admission to casualty, she developed one episode of generalized tonic-clonic seizure and was referred to the general medicine department. She also had a similar episode 1 year ago which was treated symptomatically. The patient had tachycardia of 130 per min and blood pressure of about 150 over $80 \mathrm{mmHg}$. Other vitals were within normal limits. The patient also had sensory complaints of numbness in the lower limbs. Skin lesions were absent. On neurological examination, fine tremor was present in the upper limb; deep tendon reflexes were absent in the both upper limb and lower limb. Cranial nerve examination was normal.

Complete blood count was within normal limits. Liver function test was characterized by mildly deranged serum glutamicoxaloacetic transaminase (127 IU/L) and serum glutamic pyruvic transaminase (67 IU/L). Computed tomography head plain and chest X-ray were normal. The patient had severe hyponatremia of about sodium $114 \mathrm{mEq} / \mathrm{L}$. Surgical causes were ruled out by imaging. Electromyography and nerve conduction velocity were done and found normal.

SIADH was confirmed by euvolemic hyponatremia, decreased serum osmolarity $(270 \mathrm{mOsm} / \mathrm{Kg} \mathrm{H} 20)$, increased urine sodium $(180 \mathrm{mmol} / \mathrm{L})$, increased urine osmolality (424 $\mathrm{mOsm} / \mathrm{Kg} \mathrm{H} 20$ ), normal thyroid function test, and normal

Correspondence to: Dr. Tony K S, Kizhakkemuriyil House, Chamampathal PO, Vazhoor, Kottayam, Kerala - 686517, India. E-mail: scmotodi@gmail.com

(C) 2021 Creative Commons Attribution-NonCommercial 4.0 International License (CC BY-NC-ND 4.0). 


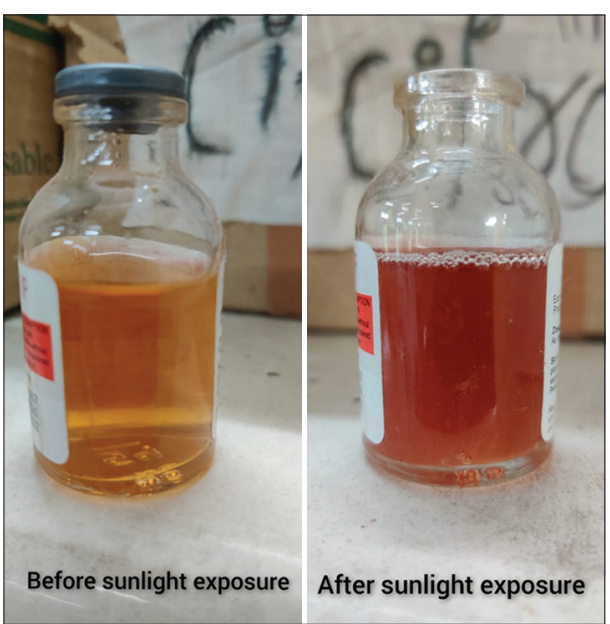

Figure 1: Classic darkening of urine on sunlight exposure (the right side showing fresh urine sample and the left side showing dark discoloration of urine after sunlight exposure)

cortisol level. Fresh urine was collected and kept in sunlight, and it demonstrated color changes on exposure to sunlight as shown in Fig. 1. Because of neurovisceral manifestations, SIADH, and the above-mentioned color changes in urine, a probable diagnosis of AIP was kept. Urine PBG was tested and was positive.

The patient was managed symptomatically and was given hydration with fluids containing glucose. Seizure which was treated with Levetiracetam $500 \mathrm{mg}$ IV stat and hyponatremia was corrected by hypertonic saline. Hyponatremia gradually improved. Hypertension and tachycardia were subsided after the acute attack. The patient became symptomatically better and was discharged with advice to avoid triggers.

\section{DISCUSSION}

Although AIP is having autosomal dominant inheritance (due to gene mutation on chromosome 11q23.3), it has weak penetrance and may skip generations [5] and because of variable manifestations, family history may not be present. Our patient had no similar disease in her family. Among the four common acute porphyrias, AIP is the most common with an incidence of about 5/100,000 people [5]. Females are more commonly affected than males. They usually present in their second through fourth decades of life [6].

Attacks usually start after puberty. Alcohol ingestion, infection, surgical procedure, variety of drugs (barbiturates, phenytoin, nifedipine, and sulfa), hypocaloric, and low-carbohydrate diets or fasting, stress, and menstruation can precipitate an acute attack. Women tend to experience flares during the luteal (progesterone) phase of the menstrual cycle $[5,7,8]$. Our patient had her symptoms initial phase of the menstrual cycle.

AIP commonly manifests in the third and fourth decades of life [3]. An acute attack is characterized by severe acute abdominal pain and neuropsychiatric symptoms. The most common symptoms are abdominal pain, nausea, vomiting, and constipation. Abdominal pain is due to severe intestinal spasms caused by autonomic dysfunction and associated with signs of increased sympathetic activity such as hypertension and tachycardia. Abdominal pain without neurological symptoms may simulate acute surgical abdomen leading to an unwanted laparotomy. Attacks may be complicated by neurologic findings (mental changes, seizure, and peripheral neuropathy that may progress to respiratory paralysis) and hyponatremia. Seizures are characteristic of severe attacks occurring only in $15-25 \%$ of cases [8]. Seizures can be attributed to several causes such as severe hyponatremia, or, less commonly, due to posterior reversible encephalopathy syndrome. Our patient had seizures. It may also develop rapidly progressive flaccid paralysis which may involve predominantly lower limbs or upper extremities or can involve all four limbs. It can cause predominantly proximal or distal weakness. Cranial nerve involvement is present in $50 \%$ of cases. Sensory loss can also occur but objective loss is rare. Mental disturbances (caused by the raised PBG and ALA) include anxiety, disorientation or hallucinations, and psychosis rarely $[1,9]$.

The most frequent presenting signs in patients with AIP are tachycardia, dark urine, confusion, peripheral motor deficit, low or absent deep tendon reflex, cranial neuropathy, and sensory loss. Oxidation of PBG to uroporphyrin and porphobilin causes purple discoloration of urine [9]. The white blood cell count may be elevated because of stress or infection. Liver enzymes will be mildly elevated during an attack which was raised in our case also. Marked hyponatremia, which is the most common electrolyte imbalance in patients with neurologic diseases, frequently complicates $40 \%$ of acute porphyrias. Its mechanism is not fully understood, usually multifactorial due to SIADH, gastrointestinal losses, infusions of hypotonic dextrose-containing fluids, nephrotoxicity, excess renal sodium excretion, and damage to the supraoptic nuclei of the hypothalamus, has been implicated in its pathogenesis. Most individuals have one or a few attacks, but 5\% of patients mainly females have recurrent attacks (defined as $>4$ attacks/year) that may persist for years.

The clue to a diagnosis of acute porphyria is red urine and hyponatremia along with neurovisceral manifestation and triggers. Despite AIP's complexity, initial diagnostic testing is simple: A urinary PBG level obtained during an attack is virtually $100 \%$ sensitive and specific for AIP and two other acute porphyrias: HCP and VP. In our patient, the diagnosis of AIP was based on clinical and urinary findings. Early diagnosis is essential to avoid progression of attacks to severe neurological complications such as motor paralysis and to institute early effective therapy $[1,9]$.

Treatment strategy during acute exacerbations includes the elimination of exacerbating factors and hospitalization in severe cases to provide supportive measures and to administer IV heme or glucose treatment. Severe tachycardia, hypertension, and arrhythmia may be treated with beta-blockers. Respiratory muscle paralysis should be managed with mechanical ventilation in an Intensive care units setting. Metoclopramide should be avoided as an antiemetic. Narcotics are safe for pain management, but barbiturates should be avoided. Hyponatremia from SIADH 
should be corrected with either fluid restriction or infusion of hypertonic saline in severe cases but with a maximum correction rate of $12 \mathrm{mmol} / \mathrm{L}$ of sodium per day. Overcorrection can lead to central pontine myelinolysis. Most anti-seizure medications actually exacerbate porphyria attacks; however, benzodiazepines and gabapentin can be used relatively safely for the treatment of prolonged seizure activity $[5,10]$. Intravenous heme suppresses the hepatic heme biosynthesis pathway through negative inhibition, decreases toxic precursors, and may decrease symptom severity and decrease the duration of hospital stay [10]. However, heme preparations can cause thrombophlebitis, transient coagulopathy, and anaphylactic reactions. Intravenous glucose is less effective than heme and is recommended only for mild attacks. Because of non-availability of heme injection, we were not able to give heme to our patient.

\section{CONCLUSION}

Diagnosis AIP requires a high clinical suspicion. This case report is a reminder to consider this rare entity as a differential diagnosis in case of the constellation of symptoms with severe abdominal pain with negative imaging along with urine discoloration following a trigger, neurological symptoms, hyponatremia, and a family history of porphyria especially in a young female should prompt immediate urine PBG testing. Early treatment should be instituted and also the patient should be given proper instructions to avoid triggers to prevent recurrent attacks.

\section{REFERENCES}

1. Ramanujam VM, Anderson KE. Porphyria diagnostics-part 1: A brief overview of the porphyrias. Curr Protoc Hum Genet 2015;86:17.20.1-26.

2. Yuan T, Li YH, Wang X, Gong FY, Wu XY, Fu Y, et al. Acute intermittent porphyria: A diagnostic challenge for endocrinologist. Chin Med J (Engl) 2015;128:1980-1.

3. Varshney G, Saini P, Ghure U. A rare case of acute intermittent porphyria with ichthyosis vulgaris in a young boy. J Fam Med Prim Care 2018;7:261.

4. Singh P, Rawat R, Zafar K. Acute intermittent porphyria with syndrome of inappropriate antidiuretic hormone secretion (SIADH) and neurological crisis, successfully treated with haemodialysis. Int J Res Med Sci 2014;2:795.

5. Dajer A, Cooper L. Acute intermittent porphyria. Emerg Med 2016;48:123-5.

6. Wheeler J. Severe acute hyponatremia as an initial presentation of acute intermittent porphyria triggered by a subdermal etonogestrel implant. Nephrol Ren Ther 2016;2:1-4.

7. Zakharova EV. Three cases of acute porphyria, presenting with severe hyponatremia. Urol Nephrol Open Access J 2015;2:55-9.

8. Singh NK, Singh DS, Singh R, Srivastava PK. Acute intermittent porphyria (a report of two cases). J Postgrad Med 1984;30:244-6.

9. Edel Y, Mamet R. Porphyria: What is it and who should be evaluated? Rambam Maimonides Med J 2018;9:e0013.

10. Wang JG, Guarnaccia M, Weiss SF, Sauer MV, Choi JM. Initial presentation of undiagnosed acute intermittent porphyria as a rare complication of ovulation induction. Fertil Steril 2006;86:462.e1-3.

Funding: None; Conflicts of interest: None Stated.

How to cite this article: Tony KS, Joshi R, Chaudhari P, Abraham R, Joshi N, Mulye A, et al. SIADH as the initial manifestation of acute intermittent porphyria: A case report. Indian J Case Reports. 2021;7(11):494-496. 